\title{
Blended learning, más allá de la clase presencial
}

\section{Blended learning, beyond the classroom}

\author{
Jesús Salinas Ibáñez \\ Bárbara de Benito Crosetti \\ Adolfina Pérez Garcías \\ Universitat de les Illes Balear, UIB (España) \\ Mercè Gisbert Cervera \\ Universitat Rovira i Virgili, URV (España)
}

\section{Resumen}

En este artículo realizamos una revisión en profundidad de la literatura de diferentes análisis y meta-análisis producidos en los últimos años para definir el concepto de e-learning y su incidencia en el ámbito universitario. A partir de los modelos, las herramientas y las estrategias que pueden aplicarse en la Educación Superior desde la perspectiva del profesorado, del alumnado o de la planificación del proceso de enseñanza-aprendizaje, ofrecemos una serie de recomendaciones orientadas a la planificación pedagógica y la integración de diferentes modalidades de formación (e-learning, b-learning y presencial), teniendo en cuenta la necesidad de orientar los procesos de formación a partir de la necesidad de flexibilidad y cambio continuo. Abordamos el b-learning desde una perspectiva general orientada a la planificación de su uso en la Educación Superior como una estrategia de cambio de carácter organizativo.

Palabras clave: blended-learning; e-learning; educación presencial; aula; universidad.

\begin{abstract}
In this paper we present a thorough literature review of different analyses and metaanalyses recently made to define the concept of e-learning and its impact on university. We consider the models, tools and strategies that can be implemented in Higher Education from the perspective of teachers, students or the teaching and learning planning process, and offer recommendations oriented to pedagogical planning and the integration of different training modalities (e-learning, b-learning and face-to-face), taking into account the need to guide training processes based on flexibility and continuous change. We approach b-learning from a general perspective oriented to plan its implementation in Higher Education as a strategy of change with an organizational character.
\end{abstract}

Keywords: blended-learning; e-learning; face-to-face learning; classroom; university. 


\section{¿QUÉ ES EL BLENDED LEARNING? UNA REVISIÓN DESDE LA INVESTIGACIÓN EDUCATIVA}

El concepto blended, desde su aparición, ha tenido diferentes denominaciones: aprendizaje híbrido, semipresencial, mixto y mezclado (Bartolomé, 2004; Llorente, 2009; Moran, 2012; Picciano, 2014). Y también visiones o significados distintos (Tayebinik y Puteh, 2012): a) blended-learning (b-learning a partir de ahora) como combinación de aprendizaje presencial-online; b) b-learning como combinación de sistemas de distribución o tecnologías de distribución de formación; c) b-learning como combinación de estrategias o modelos de aprendizaje. La primera de las tendencias se ha consolidado y es ampliamente aceptada, en cambio la terminología sigue siendo difusa y se utilizan los términos: híbrido, mixto y blended de manera intercambiable.

El término «b-learning» según Bartolomé (2004, p. 11) ha seguido "la tendencia procedente del campo de la Psicología escolar en la que destaca el término «aprendizaje» como contrapuesto al de «enseñanza»", recordándonos el autor que el aprendizaje no lo puede diseñar el docente sino que es una actividad propia del alumno. Alude a la convergencia entre dos entornos de aprendizaje, el entorno presencial, con una larga tradición en nuestros sistemas educativos, y el entorno virtual, que amplía y modifica las posibilidades de comunicación e interacción que también recoge (Graham, 2013) con posterioridad. Tradicionalmente, presencial y a distancia han sido los dos grandes sistemas formativos opuestos.

Graham (2006, p. 5) afirma que los sistemas blended learning combinan sistemas presenciales con instrucción mediada por ordenador, donde el uso de las tecnologías digitales de la comunicación e interacción en red, a tiempo real o diferido definen modelos de aprendizaje b-learning. Son sistemas "basados en la intersección entre estas modalidades que tratan de aprovecharse tanto de las ventajas y riqueza de recursos del aprendizaje virtual como de la interacción y las sinergias generadas en los grupos en las sesiones presenciales" (ELd, 2012), que para algunos (Grahan, 2006) configuran un nuevo modelo superpuesto en el que no existen diferencias pedagógicas sustanciales entre los modelos presencial y a distancia, un continuum educativo, sin clara diferenciación entre procesos docentes presenciales y virtuales .

\section{Tipologías de b-learning}

Graham (2006, 2013), Graham, Henrie y Gibbons (2014), Horn y Staker (2011), Salinas, Pérez, Darder, Orell y Negre (2008) o Staker y Horn (2012), aportan tipologías o modelos de b-learning que responden a criterios de distribución de las actividades entre el entorno presencial y a distancia o ámbitos de implementación, que aportan patrones de uso actuales del b-learning en la educación. No se han encontrado tipologías basadas en teorías o modelos de aprendizaje. 
Graham $(2006,2013)$ diferencia 4 tipologías de acuerdo con los niveles de implementación de b-learning:

1. A nivel de actividad. Una actividad de aprendizaje b-learning combina elementos presenciales con elementos basados en la comunicación mediada por ordenador.

2. A nivel de curso. Un curso b-learning combina actividades presenciales con actividades en el aula virtual, la organización temporal de estos bloques del curso puede presentarse superpuesta en el tiempo o secuenciada.

3. A nivel de programa formativo. En un programa b-learning parte del programa se realiza en línea, por ejemplo, la parte inicial y final del programa o combinando la actividad en línea con actividades de prácticas u ofreciendo itinerarios en línea para alumnos con escasa presencialidad.

4. A nivel institucional. Disponer de un modelo institucional b-learning, en algunas universidades, consiste solo en aplicar una reducción al horario presencial, en organizar un periodo del plan de estudios, un semestre, en línea; ofrecer itinerarios en línea.

Tomando como referencia a Horn y Staker (2011) y Graham et al. (2014), podemos hablar de combinación de modalidades de distribución de medios, de combinación de métodos didácticos o la combinación de instrucción cara-a-cara con instrucción en línea. A partir de éstos, se definen grupos de modelos de b-learning (figura 1):

A. De rotación. Los alumnos rotan entre modalidades de aprendizaje ya sea siguiendo una secuencia fija, a discreción del profesor, donde una de ellas es el aprendizaje en línea, mientras otras pueden incluir actividades de pequeño grupo, grupo clase, proyectos en grupo, tutorías individuales o tareas de lápiz y papel. Esta rotación puede dar lugar a distintos tipos:

- Rotación de clase o de sitio. El alumno va rotando por varias de estas actividades dentro de la misma clase, una de las cuales es aprendizaje online. Todos los alumnos pasan por todas las actividades.

- Rotación de laboratorio. En la rotación se incluye laboratorio para el aprendizaje online y la clase para otro tipo de actividades.

- Aula invertida. Aquí la rotación se da entre prácticas o proyectos guiados por el profesor cara-a-cara (durante el horario escolar) y el acceso a los contenidos y recursos del tema distribuidos online (predominantemente desde el hogar) fuera del horario escolar.

- Rotación individual. El profesor (o un algoritmo) organiza el programa de trabajo. No todos los alumnos pasan por cada uno de los sitios o cada modalidad. 
B. Flexibles. Aquí el aprendizaje en línea es la columna vertebral del proceso de formación, incluso dirige a los alumnos a realizar actividades fuera de línea. Los estudiantes cambian de modalidad de forma personalizada. Pueden tener más o menos apoyo presencial y puede dar lugar a:

- Modelo flexible. Cuando existe un cambio fluido entre modalidades y entre profesores ya sean en línea o en el campus. El apoyo online y presencial es proporcionado en función de las necesidades a partir de una base flexible y adaptativa a través de actividades de pequeño grupo, proyectos de grupo o tutoría individual. La proporción entre las actividades cara-a-cara y online es variable.

- Modelo a la carta. Los estudiantes toman uno o más cursos enteramente online con profesor remoto y al mismo tiempo continúan teniendo experiencias en el campus presencial. Pueden tomar los cursos en línea, ya sea en el campus presencial o fuera de él. Se trata de experiencias curso a curso.

- Modelo virtual enriquecido. El estudiante distribuye su tiempo entre asistir al campus presencial y el aprendizaje remoto utilizando la distribución de contenido y actividades online. Generalmente son programas online que proporcionan experiencias en el campus presencial. Difiere de la flipped classroom porque en este caso los alumnos difícilmente acuden al campus todos los días de la semana. Se trata de una experiencia escolar completa, no curso a curso como en el caso del anterior modelo (a la carta).

Figura 1. Modelos de b-learning

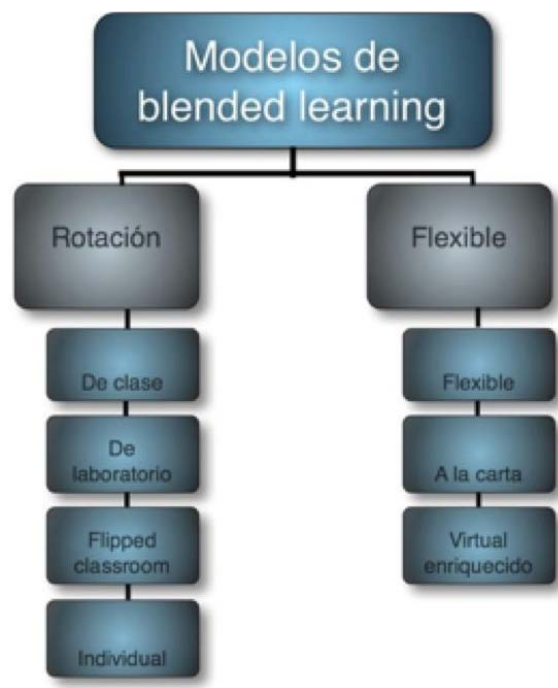

Fuente: Salinas, Darder y de Benito, 2015, p.159, tomando como referencia a Horn y Staker (2011) y Graham et al. (2014) 
Estos modelos, en general, corresponden a la modalidad formativa que han estado desarrollando las universidades y centros de formación superior. Desde que comienzan a integrarse los modelos en línea, a mediados de los años 90, se incorporan en el proceso de enseñanza-aprendizaje aspectos de la educación a distancia que favorecen los modelos mixtos.

\section{¿Qué aporta el b-Learning?}

El desarrollo del b-learning no ha venido como un modelo de aprendizaje basado en una teoría general de aprendizaje sino de la aplicación de un pensamiento ecléctico y práctico (Bartolomé, 2004 p. 13). En la actualidad, a pesar del desarrollo del b-learning y la integración de nuevos diferentes dispositivos tecnológicos móviles para facilitar la interconexión, sigue sin haber un cuerpo teórico que aporte orientación a docentes o administradores sobre cómo organizar el b-learning de manera efectiva para la mejora de los resultados de aprendizaje.

El b-learning aporta a la enseñanza la flexibilidad en los tiempos y espacios educativos, acceso a multiplicidad de recursos además de los ofrecidos por el docente, nuevos modos de interacción entre alumno-docente y entre los alumnos, incremento de la autonomía y responsabilidad del estudiante en su propio proceso (Adell y Area, 2009) como elementos de mejora educativa, además de facilitar el desarrollo de la competencia digital.

El desarrollo del b-learning ha respondido a criterios de mejora educativa y criterios económicos. Los motivos por los que se llega a modelos de b-learning son tres: 1) mejorar la educación, 2) incrementar el acceso y flexibilidad y 3) incrementar la relación coste-eficacia (Graham, 2013). Tanto si se parte del modelo de enseñanza presencial como de un modelo de enseñanza a distancia, existen dos aproximaciones al b-learning, una de corte economicista cuyo objetivo es reducir costes en personal docente y otra educativa cuyo interés es mejorar los resultados de aprendizaje, propuestas que más que excluyentes deben ser complementarias.

En parte, el desarrollo del b-learning se justifica desde los puntos débiles del e-learning. La oferta formativa en línea no siempre responde a inquietudes de mejora educativas y las instituciones, siguiendo con la visión mercantilista de la enseñanza en línea otorgan a la docencia en línea peores condiciones laborales y económicas que a la presencial. Bartolomé (2004) describe las dificultades del e-learning en torno a 1) las propias de un modelo subyacente centrado en la distribución de contenidos y 2) los defectos generados por un economicismo dominante en muchos proyectos. 


\section{Evidencias de la efectividad de b-learning según los estudios}

A medida que el concepto de b-learning ha ido ganando fuerza, se han desarrollado numerosas investigaciones dirigidas a conceptualizar el modelo y/o reportar indicadores sobre los beneficios asociados a éste.

Muestra del interés y producción científica en el tema es la cantidad de revisiones sistemáticas y meta-análisis de la investigación en b-learning. Entre estas, Siemens et al. (2015), Güzer y Caner (2014), Tayebinik y Puteh (2012), Means, Toyama, Murphy, Bakia y Jones (2009) reportan resultados positivos sobre la efectividad de modelos b-learning e identifican deficiencias, que resumimos a continuación. Estos estudios ponen de manifiesto 1) la efectividad del b-learning como modalidad de enseñanza, pero 2) la investigación no aporta información sobre los atributos educativos y, 3) apuntan a la necesidad de estructurar una pedagogía del blended como también señalan Graham et al., 2014.

Gran parte de la investigación en b-learning se ha dedicado a estudiar su efectividad en relación al modelo presencial y al totalmente a distancia. Esta investigación, inicial, aporta información sobre el impacto de este modelo y parte de su interés está en mostrar la relación coste-eficiencia del b-learning como un modelo alternativo (Siemens et al., 2015). Los meta-análisis de Means et al. (2009) y Siemens et al. (2015) reportan la existencia de diferencias significativas en los resultados de aprendizaje comparativos entre b-learning, online y presencial. Igualmente los trabajos de Graham et al. (2014), Tayebinik y Puteh (2012) y Güzer y Caner (2014) aluden a estudios que muestran la efectividad del b-learning; aportando la satisfacción de los estudiantes con el modelo, por ejemplo Güzer y Caner (2014) indican en los estudios analizados que los estudiantes valoran el b-leaning como un sistema útil, agradable, de apoyo, flexible y motivador y reportan resultados de estudios en los que la efectividad es mayor, basándose en indicadores como la satisfacción, motivación, tasa de abandono, actitudes, retención de información, etc.

Los estudios comparativos sobre la efectividad del modelo b-learning aquejan de falta de consistencia en los resultados que comparan y en lo que se entiende por b-learning, aspectos que hacen difícil la generalización de los resultados. Así mismo, este tipo de estudios requiere considerar variables como el tiempo invertido en el proceso formativo, los recursos de aprendizaje, los elementos de comunicación e interacción entre los participantes y relacionar estos como variables para explicar qué condiciones del b-learning tienen un efecto significativo en el rendimiento (Siemens et al., 2015)

Otras investigaciones, han reportado resultados sobre los beneficios delb-learning en aspectos específicos (Güzer y Caner, 2014), como puede ser el uso de la tecnología, la interacción entre los alumnos, el modelo de tutoría, la retroalimentación, qué tipo de actividades educativas son más efectivas, consideraciones pedagógicas para 
la enseñanza en b-learning, diseño de cursos, apoyo al estudiante, evaluación, rol del docente, etc. Los resultados aportados por Siemens et al. (2015) indican que estos estudios se centran en el análisis de los beneficios en relación con los modelos presencial y a distancia; por ejemplo, especifican que el estudio en diseño se proyecta a aprovechar el potencial de mejora en relación a otro modelo por separado, a cómo mejorar la presencia social y a la construcción de relaciones con la incorporación de sesiones presenciales, o a mejorar la flexibilidad de acceso o autorregulación.

Graham et al. (2014), indican que las investigaciones en diseño en b-learning se han dedicado a estudiar lo que denominan la capa física (sistema de distribución de la información o presentación de contenidos) en el coste-efectividad y accesibilidad. Pero han aportado poca información sobre los atributos del aprendizaje, la capa pedagógica o la estrategia que permite el aprendizaje.

\section{EL PAPEL DEL B-LEARNING EN LA EDUCACIÓN SUPERIOR}

Analizado en el contexto de la educación superior, el b-learning puede entenderse como un proceso de educación formal en el que el estudiante aprende "en parte, a través del aprendizaje on-line (con algún elemento de control del estudiante a través del tiempo, el lugar, la ruta y / o el ritmo) y en parte, en un lugar físico del campus con algún tipo de supervisión, y que estas distintas modalidades a lo largo del itinerario de aprendizaje en un curso o materia se interconectan para proporcionar una experiencia de aprendizaje integrado" (Salinas, Darder y de Benito, 2015, p.157). Así, puede decirse que cualquier experiencia de aprendizaje en la que el profesor incorpora un entorno virtual puede catalogarse como b-learning.

Las perspectivas, y consecuentemente los retos, que el b-learning presenta en la educación superior dependen del nivel en que ocurra la combinación: nivel de actividad (actividades de aprendizaje que contienen elementos tanto cara-a-cara como mediados por TIC), de curso, de programa o a nivel institucional (Graham, 2006).

Ocurra en el nivel que ocurra, los retos que el b-learning presenta desde la perspectiva pedagógica aparecen en la creación del nuevo espacio de aprendizaje (la metáfora del lugar de estudio que se crea mediante las TIC), en el manejo del tiempo (sobre todo por los condicionantes del entorno virtual de aprendizaje) y en la función de los encuentros cara a cara respecto al uso del entorno virtual (asociados al rol que desempeña el profesor):

- La metáfora del lugar de estudio adquiere importancia desde el momento en que las tecnologías digitales van haciendo cada vez más compleja la combinación de actividad en el campus y fuera del campus, impactando en los enfoques tradicionales de enseñanza utilizados preferentemente en las actividades oncampus y nuevas fórmulas en las actividades off-campus. Aquí concurren otros aspectos que están relacionados con el nivel de competencia digital del 
profesorado y el alumnado, que agrandan o achican la distancia transaccional y, en consecuencia, afectan a la calidad de la experiencia de aprendizaje. El concepto de distancia, al menos en relación a la comunicación, deja de ser exclusivamente geográfico.

- El tema del tiempo, de los segmentos de tiempo, que resultan un elemento crucial de organización de la enseñanza presencial, debe ser adaptado al no ser necesaria la sincronía en todas las actividades b-learning. De hecho, el manejo del tiempo sufre una profunda transformación al no poder organizarse con la misma lógica que en el formato cara-a-cara. La organización del tiempo en el b-learning supone uno de los elementos clave de la flexibilización del proceso de enseñanza-aprendizaje. La sincronía de la comunicación en los nuevos sistemas pierde nitidez con el avance de las posibilidades de los dispositivos móviles, de sistemas expertos y las de simulación de la interacción.

- Otro de los elementos clave que sustenta un entorno b-learning es el alcance y la naturaleza de los canales de comunicación que proporcionan apoyo a los estudiantes, que además de contribuir a la construcción de estos nuevos espacios y organización de los tiempos, suponen la vía de acceso a los principales recursos de aprendizaje y al espacio de interacción didáctica. Resulta, entonces, necesaria la apropiación de ese entorno de formación, la acomodación al espacio de comunicación y esto requiere, entre otras cosas: desarrollo de la competencia digital y, sobre todo, comunicativa por parte de los usuarios (docentes y estudiantes), y un fuerte apoyo y guía para la adecuada percepción de un entorno de comunicación en constante evolución.

En cuanto al binomio encuentros cara-a-cara/trabajo en el entorno virtual viene a ser el elemento diferenciador de los distintos tipos y posibilidades que presenta el b-learning, al menos a nivel institucional, de programa y de curso. En la línea de los modelos propuestos por Horn y Staker (2011) y Graham et al. (2014), mencionados más arriba, Salinas et al. (2008) establecen una tipología basada en el uso que los profesores hacen del entorno virtual, definiendo hasta 18 perfiles de profesores en cuanto a la actividad desarrollada en el campus virtual y otros aspectos didácticos (estrategia, materiales, actividades, agrupamiento, etc). Teniendo en cuenta la metodología, tanto en la parte presencial como en la virtual, los perfiles docentes pueden agruparse en seis:

1. PRESENCIAL: el peso de la asignatura está en la parte presencial, se realizan actividades, exposición didáctica, etc. de forma presencial. La plataforma educativa se utiliza para la distribución de material.

2. COMPLEMENTARIO: al igual que el perfil presencial, el peso se encuentra en la parte presencial. En cambio, la virtual se utiliza para la distribución de materiales y para que los alumnos realicen alguna actividad puntual de forma voluntaria y/o para la entrega de las actividades que se realizan de forma presencial. 
3. SUPERPUESTO: en este perfil, aunque el peso está básicamente en la parte presencial, la virtual es un complemento a la presencial, en la que se distribuye material y se realizan actividades.

4. ALTERNO: en este, entre un 50 y un $70 \%$ de la asignatura se realiza de forma presencial y el resto virtual. También se realizan actividades y se distribuye materiales de forma virtual. El rasgo que le caracteriza es que hay una separación entre la parte virtual y la presencial, que puede ser temporal o en función del tipo de actividades.

5. INTEGRADO: al igual que el alterno, entre un 50 y un $70 \%$ de la asignatura se realiza de forma presencial y el resto virtual. También se realizan actividades y se distribuye materiales de forma virtual. La diferencia se encuentra en que no hay una separación entre la parte virtual y la presencial, ambas están integradas.

6. VIRTUAL: los profesores y profesoras que se encuentran dentro de este perfil son aquellos que realizan sus asignaturas completamente de forma virtual.

Graham (2006), señala tres razones, ya mencionadas, que convierten el b-learning en atractivo para la educación superior:

- mejora pedagógica (prácticas pedagógicas más efectivas);

- incremento del acceso y la flexibilidad e

- incremento del coste-efectividad.

Estas tres razones han marcado y, en algunos casos, diferenciado los distintos proyectos universitarios de b-learning. Si la primera, la mejora pedagógica, aparece al menos nominalmente en las propuestas de las universidades, lo que sí es cierto que una mayor flexibilización en el acceso a la formación, y sobre todo, mejores ratios coste-beneficio han sido constantes en dichas propuestas.

La necesidad creciente, entre las universidades europeas, de sistemas de enseñanza-aprendizaje más flexibles y accesibles, menos costosos y a los que pueda incorporarse cualquier ciudadano a lo largo de la vida. Como dice Salinas (2008), para responder a estos retos hay que revisar los referentes actuales y promover experiencias innovadoras en los procesos de enseñanza-aprendizaje. La aplicación de las TIC a acciones de formación bajo la concepción de enseñanza flexible apunta a diversos frentes de cambio y renovación a considerar:

- Cambios en las concepciones (cómo funciona en el aula, definición de los procesos didácticos, identidad del docente, etc.).

- Cambios en los recursos básicos: Contenidos (materiales, etc.), infraestructuras (acceso a redes, etc.), uso abierto de estos recursos (manipulables por el profesor, por el alumno, etc.)

- Cambios en las prácticas del profesorado y del alumnado. 
Para ello, es fundamental una variedad de tecnologías de la comunicación que proporcionen la flexibilidad necesaria para cubrir requerimientos individuales y sociales, lograr entornos de aprendizaje efectivos, y mejorar la interacción profesoralumno. Se trata de incorporar un sentido de diseño nuevo que oriente hacia modelos de formación donde además de combinar sistemas de distribución, se incorporan estrategias didácticas centradas en el alumno, combinan lo formal y lo no formal, etc.

Los nuevos espacios educativos propiciados por el b-learning pueden referirse tanto al papel de las TIC en la enseñanza convencional como a la configuración de nuevos escenarios para el aprendizaje. Entre el aula convencional y las posibilidades de acceso a materiales de aprendizaje desde cualquier punto a través de redes de comunicación existe todo un abanico de posibilidades de acceso a recursos de aprendizaje y de establecer comunicación educativa que deben ser consideradas, sobre todo, en una proyección de futuro. Y esto también puede considerarse b-learning. Este es uno de los retos que debemos abordar en las instituciones de Educación Superior a partir de la configuración de los ecosistemas TIC y del diseño de estrategias organizativas que tengan en cuenta todos estos aspectos. Abordaremos lo que suponen todos estos retos en los siguientes apartados.

\section{ESPACIOS Y HERRAMIENTAS EN ENTORNOS B-LEARNING}

Cuando el b-learning se apoya en la tecnología tanto para la enseñanza presencial como la no presencial, se configuran diferentes espacios y herramientas que ayudan a la organización y puesta en marcha de las estrategias didácticas. Salinas et al. (2008) diferencian tres espacios que configuran los ecosistemas tecnológicos: de distribución de materiales y recursos de aprendizaje (guías, contenidos, actividades, etc.); de comunicación (profesor-alumno/s o alumno-alumno) que den apoyo a la tutoría, a la comunicación social y al proceso didáctico; y, de trabajo en grupo.

Tanto el e-learning como el b-learning son modelos que, si se aprovecha su potencialidad, pueden desarrollar importantes habilidades en el alumno para su formación como ciudadano y para su desarrollo profesional. Para ello habría que superar modelos que tratan de adaptar el modelo de enseñanza presencial a un formato web para ir a modelos donde los estudiantes se organizan en grupos y aprenden interactuando entre ellos y utilizando una vasta cantidad de recursos existentes, y el profesor actúa como guía, asesor, facilitador, o cuando es requerido. En esta línea, la multitud de experiencias realizadas y las tendencias sobre el uso de la tecnología en el proceso de aprendizaje apuntan hacia modelos más flexibles, abiertos, innovadores, y la incorporación de metodologías centradas en los estudiantes (Salinas, 2012).

Estas metodologías constituyen la manifestación más importante de la flexibilización del currículo: el estudio de casos, el sistema tutorial, el aprendizaje 
basado en problemas, el aprendizaje colaborativo y otras metodologías didácticas son vistas como alternativas pedagógicas en la formación en entornos enriquecidos por la tecnología (Salinas et al., 2008).

Paralelamente, se van desarrollando herramientas y sistemas que dan soporte a las diferentes actividades, algunas diseñadas específicamente con un objetivo educativo y en otros casos se utilizan servicios o aplicaciones de Internet de uso general (figura 2).

Tal como se ha comentado, el b-learning plantea nuevas situaciones didácticas. Una de las dimensiones que integran los entornos virtuales de enseñanzaaprendizaje (EVEA) tiene que ver con el proceso de comunicación e interacción: docente-alumnos, alumno-alumno y alumno-contenido. En el diseño de estrategias didácticas se atiende, por una parte, a la estructura comunicativa (individualizada, gran grupo y colaborativa); y, por otra, a los medios (herramientas de comunicación y materiales didácticos) necesarios para configurar la situación didáctica.

En relación con el manejo de entornos tecnológicos, Wilson, Liber, Johnson, Beauvoir, Sharples y Milligan (2007) diferencian dos posiciones: una asociada a la incorporación, utilización y generalización de los entornos virtuales en las organizaciones de educación, principalmente asociado todo ello al uso de LMS (Learning Management Systems) y otra alternativa que ofrece recursos y soluciones diferentes, centrándose en el "entorno-sujeto" (Bartolomé, 2008), donde el entorno se debe adaptar al sujeto, aprovechando la evolución del software social que promueve nuevas formas de interacción y comunicación más horizontal y que generan nuevas configuraciones basadas en los entornos personales de aprendizaje, los espacios compartidos, la redes sociales, la redes personales de conocimiento o las comunidades virtuales de aprendizaje (Marín, Salinas y de Benito, 2014; Marqués, Espuny, González y Gisbert, 2011).

Este diseño alternativo del entorno configura el ecosistema tecnológico, que se caracteriza por integrar las herramientas y atiende al acceso a contenidos y recursos, espacios de comunicación, la creación de conocimiento a partir del aprendizaje autónomo, la colaboración y la evaluación. 
Figura 2. Herramientas del ecosistema tecnológico en un entorno b-learning

\begin{tabular}{|c|c|c|c|}
\hline Tipos de herramientas & Ejemplos de herramientas/aplicaciones/recursos & Función que cumplen en el entorno & Principales técnicas didácticas asociadas \\
\hline Comunicación interpersonal & $\begin{array}{l}\text { Herramientas síncronas (videoconferencia, chat, ....) } \\
\text { Herramientas asíncronas (correo, foros,...) } \\
\text { Redes sociales }\end{array}$ & $\begin{array}{l}\text { Comunicación (personal, social, académica,...) } \\
\text { Intercambio } \\
\text { Toma de decisiones } \\
\text { Reflexión }\end{array}$ & $\begin{array}{l}\text { Tutoría } \\
\text { Debate } \\
\text { Panel } \\
\text { Exposición } \\
\text { Lluvia de ideas } \\
\text { Toma de decisiones } \\
\text { Grupos de discusión }\end{array}$ \\
\hline Gestión/Organizativas & $\begin{array}{l}\text { Agenda } \\
\text { Foros } \\
\text { Gestión de usuarios } \\
\text { Gestión de proyectos }\end{array}$ & $\begin{array}{l}\text { Gestión (grupos, usuarios, recursos,..) } \\
\text { Planiticación de la secuencia didáctica } \\
\text { Organización }\end{array}$ & Puede asociarse a cualquier técnica didáctica \\
\hline Trabajo colaborativo & $\begin{array}{l}\text { Aplicaciones compartidas } \\
\text { Edición de documentos } \\
\text { Sistemas de recomendación } \\
\text { Votaciones } \\
\text { Herramientas de comunicación interpersonal } \\
\text { Herramientas de gestión/organización }\end{array}$ & $\begin{array}{l}\text { Creación de conocimiento } \\
\text { Compartir } \\
\text { Comunicación } \\
\text { Distribución } \\
\text { Curación de contenidos } \\
\text { Toma de decisiones } \\
\text { Reflexión }\end{array}$ & $\begin{array}{l}\text { Estudio de casos } \\
\text { Aprendizaje basado en problemas } \\
\text { Trabajo por proyectos } \\
\text { Investigación social }\end{array}$ \\
\hline Interacción con el contenido & $\begin{array}{l}\text { Repositorios de recursos de aprendizaje } \\
\text { Juegos serios } \\
\text { Juegos de rol } \\
\text { Realidad aumentada } \\
\text { Realidad virtual } \\
\text { Laboratorios virtuales }\end{array}$ & $\begin{array}{l}\text { Experimentación } \\
\text { Descubrimiento } \\
\text { Reflexión } \\
\text { Creación de conocimiento } \\
\text { Compartir } \\
\text { Distribución }\end{array}$ & $\begin{array}{l}\text { Aprendizaje con materiales } \\
\text { Gamificación } \\
\text { Simulaciones y juegos de rol } \\
\text { Estudio de casos } \\
\text { Aprendizaje basado en problemas } \\
\text { Trabajo por proyectos }\end{array}$ \\
\hline Evaluación & $\begin{array}{l}\text { Portafolio digital } \\
\text { Representación a través de mapas conceptuales } \\
\text { Ejercicios, test,... de evaluación y autoevaluación } \\
\text { Gestión de datos o estadísticas de acceso }\end{array}$ & $\begin{array}{l}\text { Evaluación de los aprendizajes, procesos,... } \\
\text { Autoevaluación } \\
\text { Coevaluación } \\
\text { Seguimiento } \\
\text { Revisión }\end{array}$ & $\begin{array}{l}\text { Entrega actividades } \\
\text { Presentación de trabajos } \\
\text { Diario reflexivo } \\
\text { Entrevista de evaluación } \\
\text { Autoobservación } \\
\text { Contratos de aprendizaje }\end{array}$ \\
\hline
\end{tabular}

Fuente: Benito y Salinas, 2008 y Salinas et al., 2008

\section{EL B-LEARNING COMO ESTRATEGIA EN LA EDUCACIÓN SUPERIOR. MÁS ALLÁ DEL AULA PRESENCIAL}

Los estudiantes que llegan a la universidad actualmente esperan que ésta les ofrezca "soluciones" inmediatas a sus problemas y retos de aprendizaje con la misma facilidad que discurre su vida diaria conectada a la red, de la que reciben inputs y retroalimentación continuos. Las universidades cada vez más deberán optar por diseñar cursos en línea, incluso siendo presenciales, como una opción de futuro inmediato. También deberán asumir que las propuestas formativas a distancia o b-learning pueden favorecer un cambio en su estrategia formativa, para flexibilizarla y centrarla en el estudiante a la vez que deberán adoptar nuevas estrategias de organización de la docencia basadas en entornos TIC no presenciales.

En los apartados anteriores hemos analizado por qué llegamos a plantearnos modelos b-learning en la Educación Superior y hemos visto que, en general, se llega por dos motivos:

- Flexibilizar el proceso de formación para mejorar sus resultados (Bartolomé, 2004). 
- Incrementar el coste-beneficio del proceso de formación superior (Graham, 2013).

Aunque el primer motivo no garantiza una mejora de la eficacia y de la calidad del proceso de formación, en general, y del de aprendizaje, en particular, tampoco podemos asegurar que el segundo motivo sea suficiente en términos de mejora del proceso de aprendizaje.

En este sentido, basándonos en el informe Horizon 2016 para la Educación Superior (Johnson et al., 2016), y en las tendencias a corto plazo (1 o 2 años), destacamos la importancia de los modelos b-learning para el contexto universitario desde tres perspectivas a las que le añadimos las referencias a otras fuentes que refuerzan estas ideas:

- Estudiantes: potenciar el aprendizaje mixto ofrece una experiencia de aprendizaje cohesionada y flexible, en la que el alumno cuenta con un apoyo constante, al tiempo que le posibilita aprender de manera independiente mientras colabora con sus compañeros y con sus profesores a través de más canales de comunicación (Gallardo-Echenique, Bullen y Marqués-Molias, 2016). El b-learning favorece tanto la comunicación como la autogestión del proceso de aprendizaje.

- Profesores: gracias a la integración de las herramientas digitales para generar nuevos escenarios de aprendizaje pueden tener un seguimiento de los estudiantes de manera permanente, proporcionarles retroalimentación de acuerdo a las necesidades de aprendizaje de cada uno. Pero ello implica también un cambio en el diseño instruccional de los cursos y materias que imparten desde la perspectiva de la planificación (Sharif y Gisbert, 2015) puesto que deben tener en cuenta otras estrategias de comunicación y dinamización de su proceso de enseñanza y otras herramientas para poder monitorizar y evaluar el proceso de aprendizaje de los estudiantes. Del mismo modo, favorece el desarrollo de la competencia digital del profesorado (Gisbert y Lázaro, 2015) pues éstos deben asumir la responsabilidad de desarrollar su tarea docente en entornos tecnológicos aplicando las herramientas digitales.

- Herramientas digitales: permiten crear (y recrear) los espacios y escenarios formativos ya que adoptan la forma de comunidades de práctica (Marqués et al., 2011), laboratorios virtuales (Cela, Esteve, Esteve y Gisbert, 2014), por ejemplo, que proporcionan a los alumnos la oportunidad de realizar experimentos y simulaciones eliminando posibles riesgos. Tanto la experimentación como la simulación son fundamentales en el modelo de aprendizaje mixto o de clase invertida (Clase Invertida) en tanto que favorecen que los estudiantes resuelvan problemas y puedan aplicar de manera activa el conocimiento que van adquiriendo (Palau, Gopal, Suñé y Seritjol, 2015). 
También el Informe Horizon 2017 (Adams Becker, Cummins, Davis, Freeman, Hall, y Ananthanarayanan, 2017) en sus recomendaciones a corto plazo ( 1 o 2 años) señala que las ofertas de formación para generar aprendizaje combinado son reconocidas por su flexibilidad, facilidad de acceso y por la integración de recursos tecnológicos multimedia. Señala, también, que los modelos b-learning tienen una clara incidencia en los estudiantes desde la perspectiva del fomento del pensamiento creativo, del estudio independiente y de su capacidad para que éste pueda adaptar las experiencias de aprendizaje para poder satisfacer sus necesidades individuales.

Aunque los informes Horizon, como hemos visto destacan la importancia y la incidencia positiva de los modelos b-learning, las evidencias de la investigación no siempre coinciden con estas recomendaciones. Así, Siemens et al. (2015) a partir de un proceso de meta-análisis de 339 estudios llegan a la conclusión de que no podemos afirmar con rotundidad que el método b-learning sea mejor que los métodos presenciales o a distancia en sentido "puro". Aunque sí que evidencian, pero no lo suficiente, que desde el punto de vista de la efectividad de los aprendizajes las estrategias que utilizan la formación presencial y la distancia en el mismo programa formativo tienen efectos más positivos. De todos modos, no podemos decir que las universidades hagan modificaciones en este sentido. En la última década han dedicado mucho más esfuerzo a los cambios organizativos y de estructura de las titulaciones.

Después de que las universidades europeas adaptaran sus titulaciones de grado y máster al Espacio Europeo de Educación Superior (EEES) y basaran sus proyectos formativos en el desarrollo de las competencias de los estudiantes, desde la formación superior deberíamos asumir la responsabilidad de centrar el proceso de formación en el estudiante. Siendo así deberíamos considerar, a la hora de diseñar los proyectos formativos de las titulaciones, los modelos b-learning puesto que:

- Cada vez existen más estudiantes a tiempo parcial que necesitan conciliar el trabajo y los estudios (debido a sus limitaciones económicas) con los correspondientes problemas de horarios para poder cumplir con las exigencias de la presencialidad.

- Las TIC permiten crear nuevos escenarios para el aprendizaje incorporando más flexibilidad que exige una redefinición de espacios, tiempos y metodologías de formación.

- Una de las competencias clave del siglo XXI a las que todos los informes (desde DeSeCo (OCDE, 2005)) aluden es la competencia digital. Un entorno formativo con un claro componente TIC favorece, también, la adquisición de esta competencia orientándola a las necesidades del proceso de aprendizaje.

- La competencia digital se considera una competencia clave para el aprendizaje permanente a lo largo de la vida. Desde la perspectiva de las instituciones presenciales de educación superior las estrategias b-learning son muy indicadas para poder favorecer el desarrollo de la competencia digital de los estudiantes 
mejorando así su preparación para la incorporación al mercado laboral y profesional (Esteve, Duch y Gisbert, 2014).

Con la finalidad de adaptarnos no solo a las necesidades y características de los estudiantes sino también a las necesidades del mercado laboral y profesional aparecen modelos de universidades y modelos formativos emergentes como por ejemplo las Universidades Corporativas. Éstas tienen como principales características su capacidad de diseñar y desarrollar titulaciones a medida en "tiempo real", la consideración del aula de formación desde la perspectiva del entorno personal de aprendizaje del estudiante (PLE) y la tecnología digital como principal aliada para superar las barreras del espacio y tiempo y de este modo flexibilizar tanto los escenarios como el proceso de enseñanza-aprendizaje.

\section{CONCLUSIONES}

Proponemos aquí una serie de recomendaciones a todas aquellas instituciones que se planteen adoptar un modelo b-learning para su institución. En primer lugar, creemos que es importante no empezar desde cero. Tener en consideración las buenas prácticas para poder aprovechar la experiencia de otros proyectos y no invertir más tiempo del necesario en los proyectos b-learning (Siemens et al., 2015). En segundo lugar, tener en cuenta que adoptar este tipo de modelos no implica, necesariamente, reducir las horas de presencialidad de los modelos presenciales pues la no presencia en clase del profesor y el estudiante supone la necesidad de planificar de otro modo el proceso de E-A (Sharif y Gisbert, 2015) y de utilizar, como hemos apuntado en los apartados anteriores, herramientas tecnológicas que nos permitan gestionar la parte no presencial del proceso formativo. Para ello podemos adoptar dos perspectivas organizativas, teniendo siempre al estudiante como centro, que consideramos fundamentales: la perspectiva de las actividades formativas si nos centramos en el proceso de aprendizaje (Salinas et al., 2008), y la perspectiva del estudiante (Staker y Horn, 2012) si nos centramos en las necesidades de flexibilidad, comunicación, escenarios tecnológicos para el aprendizaje de éste.

Consideramos fundamental continuar investigando las posibilidades del b-learning como estrategia para superar la visión del aula y la clase presencial como "feudo" del profesorado y las evidencias presenciales como fuente de información principal para sistematizar el proceso de aprendizaje de los estudiantes y evaluarlos. Aunque en todas las universidades se dispone de un campus virtual este sólo hecho no puede garantizar que la institución tenga una estrategia b-learning. Todos los trabajos de revisión sistemática revisados, apuntan a la necesidad de incidir, desde la investigación, en:

1. Identificar elementos clave de la capa pedagógica del diseño que provocan aprendizajes de interés, así como entender cómo atributos de diferentes capas 
se complementan y trabajan juntos. Así mismo, notan en falta estudios iterativos que permitan identificar los atributos clave del modelo y su influencia en los efectos deseados, los cuales puedan ser probados para explicar los resultados comparativos obtenidos (Graham et al., 2014).

2. Desarrollar estudios centrados en el aprendizaje digital, desarrollar informes de prácticas más detallados que permitan la comprensión de matices de b-learning más allá de la distribución combinada y enfocar la investigación en la interacción entre procesos de aprendizaje (Siemens et al., 2015).

3. Estructurar una pedagogía del blended (Graham et al., 2014). Moran (2012) considera el b-learning como una alternativa posible para la configuración de una nueva formación que trasciende los espacios del aula y se traslada a todas las esferas de la vida de las personas. En este sentido, señala una serie de aspectos que podrían incorporarse a una pedagogía del b-learning: hipermedialidad, la sincronía y asincronía, andamiaje personalizado y andamiaje colectivo, accesibilidad a los materiales, interacción.

4. Estudiar la integración de modelos constructivistas y colaborativos en entornos b-learning y animar a formar estudiantes creativos y curiosos "who reads, writes and produces for the world" (Güzer y Caner, 2014, 462).

Por último, queremos destacar la importancia de diseñary desarrollar ecosistemas TIC para facilitar el uso de estas herramientas. Un uso que, progresivamente, debe estar orientado a la gestión del proceso de aprendizaje por parte del estudiante y a la planificación tanto de los escenarios como del proceso de formación en sí.

\section{REFERENCIAS BIBLIOGRÁFICAS}

Adams Becker, S., Cummins, M., Davis, A., Freeman, A., Hall Giesinger, C., y Ananthanarayanan, V. (2017). NMC Horizon Report: 2017 Higher Education Edition. Austin, Texas: The New Media Consortium.

Adell, J., y Area M. (2009). eLearning: Enseñar y aprender en espacios virtuales. En J. De Pablos (Coord.), Tecnología Educativa. La formación del profesorado en la era de Internet. Aljibe, Málaga, 391424.

Bartolomé, A. (2004). Blended Learning. Conceptos básicos. Pixel-Bit, Revista de Medios y Educación, 23, 7-20.

Bartolomé, A. (2008). Entornos de aprendizaje mixto en Educación Superior.
RIED. Revista Iberoamericana de Educación a Distancia, 11, 15-51.

Cela, J., Esteve, V., Esteve, F., y Gisbert, M. (2014). 3D simulation as a learning environment for acquiring the skill of selfmanagement. An experience with Spanish university students of Education. Journal of Educational Computing Research, 51(3), 295-309.

de Benito, B., y Salinas, J. (2008). Los entornos tecnológicos en la universidad. Pixel-Bit, Revista de Medios y Educación, 32, 83-10o.

ELd (2012). e-Learning docs $\sim$ Recursos para formación a través de TIC. Recuperado de https://elearningdocs. wordpress.com/2012/07/22/blendedlearning 
Esteve, F., Duch, J., y Gisbert, M. (2014). Los aprendices digitales en la literatura científica: Diseño y aplicación de una revisión sistemática entre 2001 y 2010. Pixel-Bit, Revista de Medios y Educación, 45, 9-21.

Horn, M., y Staker, H. (2011). The Rise of K-12 Blended Learning. Innosight Institute.

Gallardo-Echenique, Bullen, MarquésMolías (2016). Student Communication and Study Habits of First-year University Students in the Digital Era. Canadian Journal of Learning \& Technology, 42(1).

Gisbert, M., y Lázaro, J. L. (2015). Professional development in teacher digital competence and improving school quality from the teachers' perspective: a case study. Journal of New Approaches In Educational Research, 4(2), 115-122.

Graham, C. R. (2006). Blended Learning Systems. Definition, current trends and Future Directions. En J. Curtis, Ch. Bonk y R. Graham (Ed.), The Handbook of Blended Learning: Global Perspectives, Local Designs. John Wiley \& Sons.

Graham, C. R. (2013). Emerging practice and research in blended learning. In M. G. Moore (Ed.), Handbook of distance education (3rd ed.), (333-350). New York, NY: Routledge.

Graham, C., Henrie, C., y Gibbons, A. (2014). Developing models and Theory for Blended Learning Research. En A. Picciano, C. Dziuban, y C. Graham (Eds.), Research Perspectives in Blended Learning: Research Perspectives, 2(1333). NY. P.: Routledge.

Güzer, B., y Caner, H. (2014). The Past, Present and Future of Blended Learning: An in Depth Analysis of Literature. Procedia-Social and Behavioral Sciences, 116, 4596-4603. Recuperado de http:/ doi.org/10.1016/j.sbspro.2014.01.992

Johnson, L., Adams Becker, S., Cummins, M., Estrada, V., Freeman, A., y Hall, C. (2016). NMC Horizon Report: 2016
Higher Education Edition. Austin, Texas: The New Media Consortium.

Llorente, M. C. (2009). Formación semipresencial apoyada en Red (Blended Learning). Diseño de acciones para el aprendizaje. Eduforma, Alcalá de Guadaira.

Marín, V., Salinas, J., y de Benito, B. (2014). Research results of two Personal Learning Environments experiments in a higher education institution. Interactive Learning Environments, 22(2) (205-220) doi: 10.1080/10494820.2013.788031.

Marqués, L., Espuny, C., González, J., y Gisbert, M. (2011). La creación de una comunidad aprendizaje en una experiencia de blended learning. PixelBit. Revista de Medios y Educación, 39, 55-68.

Means, B., Toyama, Y., Murphy, R., Bakia, M., y Jones, K. (2009). Evaluation of Evidence-Based Practices in Online Learning. Structure, 66. doi: http://doi. org/10.1016/j.chb.2005.10.002

Morán, L. (2012). Blended-learning. Desafío y oportunidad para la educación actual. Edutec, Revista Electrónica de Tecnología Educativa, 39. Recuperado de http:/ dx.doi.org/10.21556/edutec.2012.39.371

Organisation for Economic Co-operation and Development (OECD). (2005). The definition and selection of key competencies (DeSeCo). Executive summary. OECD.

Palau, R., Gopal, J., Suñé, X., y Seritjol, F. (2015). Ventajas y desventajas de la aplicación del flipped classroom. Comunicación y Pedagogía, 285-286.

Picciano, A. (2014). Introduction to Blended learning: research perspectives. En A. Picciano, C. R. Dziuban y C. R. Graham (Ed.), Blended learning: research perspectives.. New York and London: Routledge.

Salinas, J. (2008). Innovación educativa y uso de las TIC. En J. Salinas (Coord.), Innovación educativa y uso de las TIC. 
Sevilla: Universidad Internacional de Andalucía, (15-30).

Salinas, J. (2012). La investigación ante los desafíos de los escenarios de aprendizaje futuros. RED. Revista de Educación a Distancia, 32.

Salinas, J., Pérez, A., y de Benito, B. (2008). Metodologías centradas en el alumno para el aprendizaje en red. Madrid: Síntesis.

Salinas, J., Pérez, A., Darder, A., Orell, J. y Negre, F. (2008). Perfiles metodológicos de los profesores en procesos de enseñanza-aprendizaje en entornos virtuales. EDUTEC 2008. Las TIC, puente entre culturas: Iberoamérica y Europa. Santiago de Compostela.

Salinas, J., Darder, A. y de Benito, B. (2015). Las TIC en la enseñanza superior: e-learning, b-learning y m-learning. En J. Cabero y J. Barroso (Coord.), Nuevos retos en tecnología educativa. Madrid: Síntesis, (153-174).

Sharif, A., y Gisbert, M. (2015). The impact of culture on instructional design and quality. International Journal of Instruction, 8(1), 143-156, Turquia. ISSN 1694-609X.

Siemens, G., Gašević, D., y Dawson, S. (2015). Preparing for the digital university: a review of the history and current state of distance, blended, and online learning. Recuperado de http://linkresearchlab. org/PreparingDigitalUniversity.pdf

Staker, H., y Horn, M. B. (2012). Classifying K-12 Blended Learning. Innosight Institute, (Mav), 22. doi: http://doi. org/10.1007/s10639-007-9037-5

Tayebinik, M., y Puteh, M. (2012). Blended Learning or E-learning? International Magazine on Advances in Computer Science and Telecommunications, 3(1), 103-110. doi: http://doi.org/10.1016/j. iheduc.2012.12.001

Wilson, S., Liber, O., Johnson, M., Beauvoir, P., Sharples, P., y Milligan, C. (2007). Personal Learning Environments: Challenging the dominant design of educational systems. Je-LKS: Journal of E-Learning and Knowledge Society, 3(2).

\section{PERFIL ACADÉMICO Y PROFESIONAL DE LOS AUTORES}

Jesús Salinas Ibáñez. Doctor en Filosofía y Ciencias de la Educación. Catedrático de Didáctica y O. Escolar en la Universidad de las Islas Baleares. Coordinador del Doctorado Interuniversitario en Tecnología Educativa. Investigador principal del Grupo de Tecnología Educativa. Director de Edutec. Revista electrónica de Tecnología Educativa.

E-mail: iesus.salinas@uib.es

Bárbara de Benito Crosetti. Doctora en Ciencias de la Educación. Colaboradora doctora de Didáctica y O. Escolar en la Universidad de las Islas Baleares. Miembro de los grupos de investigación GTE y GITED de la UIB. E-mail: barbara.debenito@uib.es

Adolfina Pérez Garcies. Doctora en Ciencias de la Educación. Profesora Titular de Didáctica y O. Escolar en la Universidad de las Islas Baleares. Miembro de los grupos de investigación GTE y GITED de la UIB.

E-mail: fina.perez@uib.es 


\section{DIRECCIÓN DE LOS AUTORES}

Institut de Recerca i Innovació Educativa.

Universidad de las Islas Baleares

Edificio Sa Riera C / Miguel de los Santos Oliver, 2

07122 - Palma de Mallorca (España)

Mercè Gisbert Cervera. Doctora en Ciencias de la Educación. Profesora Titular de Didáctica y O. Escolar en la Universidad Rovira i Virgili. Investigadora principal del grupo de investigación ARGET de la URV. Coordinadora del Máster interuniversitario a distancia Tecnología Educativa: e-learning y Gestión del Conocimiento. Directora de la Revista UTE. Revista electrónica Universitas Tarraconensis. Revista de Ciencias de la Educación.

E-mail: merce.gisbert@urv.cat

\section{DIRECCIÓN DE LA AUTORA}

Departamento de Pedagogía

Universitat Rovira i Virgili

Campus Sescelades

Ctra. de Valls s/no.

Tarragona, 43007 (España)

Fecha de recepción del artículo: 26/04/2017

Fecha de aceptación del artículo: 18/07/2017

\section{Como citar este artículo:}

Gisbert Cervera, M., de Benito Crosetti, B., Pérez Garcies, A.,Salinas Ibáñez, J. (2018). Blended Learning, más allá de la clase presencial. RIED. Revista Iberoamericana de Educación a Distancia, 21(1), pp. 195-213. doi: http://dx.doi. $\operatorname{org} / 10.5944 /$ ried.21.1.18859 